\title{
On the Modelling of Inter-sub-symbol Interference in GFDM Transmission
}

\author{
Author 1 and Author 2
}

\begin{abstract}
Inter-sub-symbol interference (ISSI) refers to the interference that independently affects each group of sub-symbols transmitted on the same sub-carrier in generalized frequency division multiplexing (GFDM). In this letter we derive a model to describe ISSI when transmission takes place over a frequency selective channel. The proposed model, which is derived by exploiting the discrete Gabor transform (DGT) structure of GFDM, allows for the exact computation of the channel impulse response associated with each sub-carrier. This knowledge makes easier the design of detection algorithms both on a symbol-bysymbol basis and on a per sub-carrier basis. Minimum meansquared error and maximum likelihood receiver are considered as examples of the two different detection strategies together with the avaluation of their complexity. Simulation results demonstrate the improvement in the symbol error rate performance compared to another approach recently proposed in the literature that does not take into account the effect of ISSI.
\end{abstract}

Index Terms-Discrete Gabor transform (DGT), maximum likelihood detection, generalized frequency division multiplexing.

\section{INTRODUCTION}

In existing fourth generation (4G) cellular systems and in, almost ready, to be launched fifth generation $(5 \mathrm{G})$ wireless networks, orthogonal frequency division multiplexing (OFDM), based on discrete Fourier transform (DFT), is applied across the entire system bandwidth using a fixed set of waveform parameters [1]. A main issue of such a design is the lack of flexibility to support mixed services with different waveform parameters within one carrier, which is a key requirement in the physical layer of future cellular networks [2].

The look for new waveforms that are able to support variable and customizable pulse shaping filters is one of the research priorities in order to achieve a better trade-off between timedomain and frequency-domain localization [2]. With this aim, generalized frequency division multiplexing (GFDM), which is based on the use of circular filtering at sub-carrier level, was proposed as modulation scheme [2], [3]. Compared to OFDM, the main advantages of GFDM are a reduction of the out-of-band (OOB) emission, achieved by means of filtering at sub-carrier level [3]-[5], and in an increase of the spectral efficiency, obtained through the introduction of tail biting, which makes the length of the cyclic prefix (CP) independent from that of pulse shaping filter [6], [7]. Also, the flexible frame structure of GFDM allows, by changing the number of time slots and of sub-carriers in a frame, covering both conventional OFDM and DFT-spread OFDM (DFT-s-OFDM), which results in complete backward compatibility with 5G [6].

Despite the above mentioned advantages, a main issue of GFDM compared to OFDM is the need of equalization, implemented by block-based processing in time or frequency domain, that is required even in the case of transmission over an ideal channel [3]. Equalization is required because most of the prototype filters used for GFDM make it a non orthogonal system, introducing intercarrier interference (ICI) and intersymbol interference (ISI). Therefore, the removal of ICI and ISI requires additional processing compared to OFDM, which finally increases the complexity of the receiver [4]. For this reason, the use of prototype filters such as the Dirichlet pulse, which are both ICI- and ISI-free and that can be easily applied in GFDM with low complexity and low latency, has recently attracted increasing interest [8]. The use of these filters allows for an efficient implementation of the GFDM receiver by exploiting a relationship between the GFDM signal and the discrete Gabor transform (DGT) that was proposed in [9]. It was shown that GFDM transmission and reception are equivalent to a finite discrete Gabor expansion and DGT in critical sampling, respectively. In particular, the Dirichlet pulse shaping filter is extensively used to evaluate the performance of GFDM systems in terms of error rate and OOB emission [10], [11].

The Gabor interpretation allows for an efficient computation of the zero-forcing (ZF) and minimum mean-squared error (MMSE) receiving filters without resorting to matrix inversion. In particular, an optimal symbol-by-symbol detector can be used after the DGT-based receiver when transmisison takes place over an ideal channel [12]. An equivalent interpretation of the DGT receiver was given in [13], which allows for signal recovery with complexity lower than in time-domain.

However, it is worth observing that in case of transmission over a non-ideal channel the DGT interpretation with critical sampling looses its validity. In this case, the effect of the channel must be taken into account in the frequency-domain equalization of the whole GFDM symbol. This was considered in [13], where it was observed that the performance of the proposed low-complexity approach for GFDM in the DGT setting is close to that of OFDM only when the number of sub-symbols transmitted on each sub-carrier is low. When this number increases a worst performance is observed compared to OFDM. This degradation of performance is due to the inter-sub-symbol interference (ISSI) that arises from the subsymbols transmitted on the same sub-carrier. The effect of ISSI is not modeled in [13], where an approximate maximum likelihood (ML) detector is proposed for deciding transmitted symbols. Whereas, in this letter we propose an exact modelling of ISSI that allows for the computation of the exact channel impulse response on each sub-carrier and thus makes easier the design of detection algorithms.

\section{GFDM SySTEM MODEL}

With reference to one GFDM symbol, the block of the transmitted bits is applied to the input of a modulator that 
gives at its output an $M \times K$ data matrix $\mathbf{D}$ whose $N=M K$ elements take values from a complex constellation [7]. The data matrix $\mathbf{D}$ is sent to the GFDM modulator, based on $K$ sub-carriers, where each sub-carrier is used to transmit $M$ subsymbols. According to this model the data matrix $\mathbf{D}$ can be represented as the composition of $K$ column vectors

$$
\mathbf{D}=\left[\mathbf{X}_{0}, \mathbf{X}_{1}, \cdots, \mathbf{X}_{K-1}\right],
$$

where

$$
\mathbf{X}_{q}=\left[X_{q}(0), X_{q}(1), \cdots, X_{q}(M-1)\right]^{T},
$$

being $(\cdot)^{T}$ the transposition operation and $X_{q}(m)$ the $m$ th sub-symbol, with $m=0, \ldots, M-1$, transmitted on the $q$ th sub-carrier, with $q=0, \ldots, K-1$. The time-duration of each symbol vector $\mathbf{X}_{q}$ is $M T_{s}$ with sub-carrier spacing equal to $1 /\left(M T_{s}\right), T_{s}$ being the symbol interval on each sub-carrier. The $M$ sub-symbols of the $q$ th group are up-sampled by a factor $K$ and applied to the input of a periodic pulse shaping filter with $N$ coefficients. After pulse shape filtering, the $n$th sample of the transmitted GFDM signal is written as

$$
x(n)=\sum_{q=0}^{K-1} \sum_{m=0}^{M-1} X_{q}(m) g_{q, m}(n), \quad n=0,1, \cdots, N-1,
$$

where

$g_{q, m}(n)=g\left(\langle n-m K\rangle_{N}\right) e^{\frac{j 2 \pi q n}{K}}, m=0, \cdots, M-1, q=0, \cdots, K-1$,

are frequency-domain shiftings and cyclic time-domain translations of the discrete-time prototype impulse response $g(n)$ of length $N$, with $\langle\cdot\rangle_{N}$ denoting the modulo $N$ operation.

\section{A. Transmission over the Ideal Channel}

In case of transmission over an ideal channel the model of the received signal is given by

$$
y(n)=x(n)+w(n), \quad n=0,1, \cdots, N-1,
$$

where $w(n)$ is the zero mean complex additive white Gaussian noise (AWGN) with variance $\sigma^{2}$. In this case optimal detection can be easily implemented by exploiting the DGT structure of GFDM. In fact, as first observed in [9], (3) can be interpreted as an inverse DGT (IDGT) having $g_{q, m}(n)$ as synthesis function. According to this interpretation, symbols $X_{q}(m)$ can be recovered from $x(n)$ by applying DGT as

$$
X_{q}(m)=\sum_{n=0}^{N-1} \gamma_{q, m}^{*}(n) x(n),
$$

where $\gamma_{q, m}$ is a periodic discrete function with period $N$, which is obtained from time and frequency shifts of an analysis window $\gamma(n)$ as

$$
\gamma_{q, m}(n)=\gamma\left(\langle n-m K\rangle_{N}\right) e^{\frac{j 2 \pi q n}{K}} .
$$

Note that, the identity defined by (6) only holds when the synthesis function $g(n)$ and the analysis function $\gamma(n)$ satisfy the Wexler-Raz identity given in [13, eq. (11)]. If this condition is not satisfied interference arises both from symbols transmitted on other sub-carriers and from sub-symbols transmitted on the same sub-carrier. The optimal ML detection is obtained by computing the DGT of the received signal in (5) and applying the resulting sequence to a memoryless threshold detector.

\section{B. Transmission over the Frequency-Selective Channel}

When transmission takes place over a frequency-selective channel, the effect of inter-symbol interference introduced by the time spread of the channel can be mitigated by inserting a CP, which consists of $N_{C P}$ samples such that the length of the $\mathrm{CP}$ is at least equal to the length of the channel. The $\mathrm{CP}$-extended signal is written as

$$
\tilde{x}(n)= \begin{cases}x(N+n), & n=-N_{C P}, \ldots,-1, \\ x(n), & n=0, \ldots, N-1 .\end{cases}
$$

We consider the $L$-path tapped delay line channel model of [14] with continuous-time impulse response defined by

$$
h(t)=\sum_{i=0}^{L-1} h_{i} \delta\left(t-\tau_{i}\right),
$$

where $h_{i}$ is the complex amplitude, i.e., tap coefficient, of the $i$ th path associated with the delay $\tau_{i}$ and $\delta(t)$ is the delta Dirac function. When $L=1$ and $h_{0}=1$ we get the ideal channel. In the following, for simplicity, we consider $\tau_{i}=i$.

Considering the convolution between $\tilde{x}(n)$ in (8) and the channel in (9), the received signal is modelled as

$$
y(n)=\sum_{i=0}^{L-1} h_{i} \tilde{x}(n-i)+w(n) .
$$

Under the assumption $N_{C P} \geq L-1$, after removing the effect of the cyclic prefix and by replacing (3) in (10), we get

$y(n)=\sum_{i=0}^{L-1} h_{i} \sum_{k=0}^{K-1} \sum_{l=0}^{M-1} X_{k}(l) g(\langle n-i-l K\rangle) e^{\frac{j 2 \pi k\langle n-i\rangle}{K}}+w(n)$.

In order to recover the transmitted symbols, the DGT defined in (6) is applied to the received signal as

$$
\begin{aligned}
& Y_{q}(m)=\sum_{n=0}^{N-1} \gamma_{q, m}^{*}(n) y(n)=\sum_{n=0}^{N-1} \gamma^{*}\left(\langle n-m K\rangle_{N}\right) y(n) e^{-\frac{j 2 \pi q n}{K}} \\
= & \sum_{k=0}^{K-1 M-1} \sum_{l=0}^{L-1} X_{k}(l) \sum_{i=0}^{L-1} h_{i} P_{(q-k) M}((m-l) K, i) e^{-\frac{j 2 \pi k i}{K}}+W_{q}(m), \text { (12) }
\end{aligned}
$$

where $W_{q}(m)$ is the DGT of the AWGN and

$$
\begin{gathered}
P_{k M}(l K, i)=\sum_{n=0}^{N-1} \gamma^{*}(n) g\left(\langle n-i+l K\rangle_{N}\right) e^{-\frac{j 2 \pi k n}{K}} \\
=\frac{1}{N} \sum_{q=0}^{N-1} \Gamma_{q}^{*} G_{\langle q+k M\rangle_{N}} e^{-\frac{j 2 \pi(q+k M)(i-l K)}{N}} \\
=\left(\frac{1}{N} \sum_{q=0}^{N-1} \Gamma_{q}^{*} G_{\langle q+k M\rangle_{N}} e^{\frac{j 2 \pi q l}{M}} e^{-\frac{j 2 \pi q i}{N}}\right) e^{-\frac{j 2 \pi k i}{K}} \\
0 \leq k \leq K-1, \text { and } 0 \leq l \leq M-1,
\end{gathered}
$$

with $\Gamma_{q}$ and $G_{q}$ corresponding to the $N$-points DFT of $\gamma(n)$ and $g(n)$, respectively. In the special case $i=0,(13)$ converts converts into eq. (14) of [13]

$$
\begin{aligned}
P_{k M}(l K, 0) & =\frac{1}{N} \sum_{q=0}^{N-1} \Gamma_{q}^{*} G_{\langle q+k M\rangle_{N}} e^{\frac{j 2 \pi q l}{M}}=\delta(k) \delta(l), \\
0 & \leq k \leq K-1, \text { and } 0 \leq l \leq M-1,
\end{aligned}
$$


which expresses the condition of biorthogonality between $\Gamma_{q}$ and $G_{q}$ in a way equivalent to the Wexler-Raz identity between $\gamma(n)$ and $g(n)$.

As a function satisfying the Wexler-Raz identity with critical sampling in what follows we consider the Dirichlet function, also referred as periodic sinc, which is characterized by a DFT that is a rectangular pulse

$$
G_{k}^{D}=\left\{\begin{array}{cc}
1, & \left(0 \leq k \leq\left\lceil\frac{M}{2}\right\rceil-1\right) \cup\left(N-\left\lfloor\frac{M}{2}\right\rfloor \leq k \leq N-1\right), \\
0 & \text { otherwise, }
\end{array}\right.
$$

where $\lceil\cdot\rceil$ and $\lfloor\cdot\rfloor$ denote the nearest upper and lower integer, respectively. By using Dirichlet function it turns out that $\gamma(n)=g(n)$ and the matched and the ZF conditions are satisfied at the same time [9]. Also, as shown in the following, the Dirichelet function allows to implement parallel detection on a per sub-carrier basis, which allows to decrease latency and complexity at the same time. By setting $G_{k}=G_{k}^{D}$ in (13) we get

$$
P_{k M}(l K, i)=\delta(k) \frac{1}{N} \frac{\sin \left(\frac{\pi(i-l K)}{K}\right)}{\sin \left(\frac{\pi(i-l K)}{N}\right)} e^{\frac{j \pi(i-l K)\left(1+(-1)^{M}\right)}{2 N}},
$$

where $\delta(k)$ denotes the Kronecher delta. By substituting (16) in (12), after some mathematical manipulation, we get

$$
\begin{aligned}
Y_{q}(m) & =\sum_{l=0}^{M-1} X_{q}(l) \sum_{i=0}^{L-1} h_{i} \frac{\sin \left(\frac{\pi(i-(m-l) K)}{K}\right)}{\sin \left(\frac{\pi(i-(m-l) K)}{N}\right)} e^{\frac{-j 2 \pi q i}{K}}+W_{q}(m) \\
& =\sum_{l=0}^{M-1} X_{q}(l) \bar{H}_{q M}((m-l) K)+W_{q}(m),
\end{aligned}
$$

where the even property of Dirichlet function has been used. Equation (17) demonstrates that when the Dirichlet function is chosen as impulse response for the analysis and synthesis filters, the interference is due only to sub-symbols transmitted on the same sub-carrier while those transmitted on other subcarriers are irrelevant. As mentioned above, this allows to implement detection in each sub-carrier and, therefore, reduces the overall complexity of the receiver. The "windowed" channel in (17) is given by

$$
\bar{H}_{q}(m)=\sum_{i=0}^{N-1} h_{i}^{(Z P)} b_{\langle i-m\rangle_{N}} e^{\frac{-j 2 \pi q i}{N}},
$$

where $b_{\langle n-m\rangle_{N}}$ corresponds to a shifting of $m$ samples of the periodic function

$$
b_{n}=\frac{\sin (\pi n / K)}{\sin (\pi n / N)}, \quad n=0, \ldots, N-1,
$$

and

$$
h_{i}^{(Z P)}= \begin{cases}h_{i} & i=0, \ldots, L-1, \\ 0 & i=L, \ldots, N-1 .\end{cases}
$$

As it can be clearly observed, (18) is the DFT of the product of two discrete time periodic functions. Therefore, it can be rewritten as the periodic convolution of period $N$ of the DFTs of the two functions as

$$
\bar{H}_{q}(m)=H_{q} \otimes_{N} B_{q} e^{-j \frac{2 \pi m q}{N}},
$$

where $\otimes_{N}$ represent the periodic circular convolution, $H_{q}$ is the DFT of the zero-padded channel in (20) and $B_{q} e^{-j \frac{2 \pi m q}{N}}$ is the DFT of the shifted windowing function, with $B_{q}=G_{q}^{D}$. Since $G_{k}^{D}$ given in (15) is a rectangle of width $M$ cenetered in 0 , it turns out that (21) implements $M$ moving averages with weights that are defined by the values assumed by the complex exponential at different values of $m$.

After some straightforward mathematical manipulations, (12) can be rewritten as

$$
Y_{q}(m)=\underbrace{X_{q}(m) \bar{H}_{q M}(0)}_{\begin{array}{c}
m \text { th sample received } \\
\text { on the } q \text { th sub-carrier }
\end{array}}+\underbrace{\sum_{l=0, l \neq m}^{M-1} X_{q}(l) \bar{H}_{q M}((m-l) K)}_{\begin{array}{c}
\text { Interference induced by sub-symbols } \\
\text { other than } X_{q}(m) \text { transmitted } \\
\text { on the } q \text { th sub-carrier }
\end{array}}+\underbrace{W_{q}(m)}_{\text {AWGN Noise }},
$$

where it appears that the $m$ th sub-symbol transmitted on the $q$ th sub-carrier $X_{q}(m)$ is

- scaled by the term $\bar{H}_{q M}(0)$;

- impaired by the interference generated by the lth subsymbol transmitted on the same sub-carrier through the term $\bar{H}_{q M}(l-m) K, l \neq m$;

- distorted by the AWGN term $W_{q}(m)$.

Remark To confirm the flexibility of the GFDM system from eq. (17), the two extreme cases of GFDM system are:

- OFDM, for $M=1$, where each sub-carrier is used to transmit only one symbol and $K=N$. We get the conventional OFDM where $P_{k}(0, i)=\delta_{k}, \forall i$.

- DFT-s-OFDM, for $N=M$, that corresponds to singlecarrier transmission where $P_{0}(l, i)=\delta_{N}(i-l), \forall l, i$, which is periodic Kronecher delta with period $N$.

\section{Per Sub-CARrier Detection}

From the model defined in (22) it is possible to define the received signal vector $\mathbf{Y}_{q}=\left[Y_{q}(0), Y_{q}(1), \ldots, Y_{q}(M-1)\right]^{T}$ on the $q$ th sub-carrier as

$$
\mathbf{Y}_{q}=\overline{\mathbf{H}}_{q M} \mathbf{X}_{q}+\mathbf{W}_{q}, \quad q=1, \ldots, K-1,
$$

where $\mathbf{W}_{q}=\left[W_{q}(0), W_{q}(1), \ldots, W_{q}(M-1)\right]^{T}$ is the vector of noise samples and

$\overline{\mathbf{H}}_{q M}=\left[\begin{array}{cccc}\bar{H}_{q M}(0) & \bar{H}_{q M}(N-K) & \cdots & \bar{H}_{q M}(N-(M-1) K) \\ \bar{H}_{q M}(N-(M-1) K) & \bar{H}_{q M}(0) & & \cdots \\ \vdots & \ddots & \ddots & \bar{H}_{q M}(N-K) \\ \bar{H}_{q M}(N-K) & \bar{H}_{q M}(N-2 K) & \cdots & \bar{H}_{q M}(0)\end{array}\right]$

is the "windowed" channel matrix. The model given in (23) constitutes the basis on which to build detection algorithms for the $M$ sub-symbols transmitted on the $q$ th sub-carrier, i.e., on a per-sub-carrier basis. In order to take a decision on the transmitted symbols we consider two possible approaches:

- Symbol-by-Symbol Detection, where the receiver takes independent decisions for each symbol transmitted on a given sub-carrier. As a symbol-by-symbol receiver we consider linear MMSE, where transmitted symbols are estimated as [9]

$$
\hat{\mathbf{X}}_{q}=\mathbf{C}_{q M} \mathbf{Y}_{q} .
$$




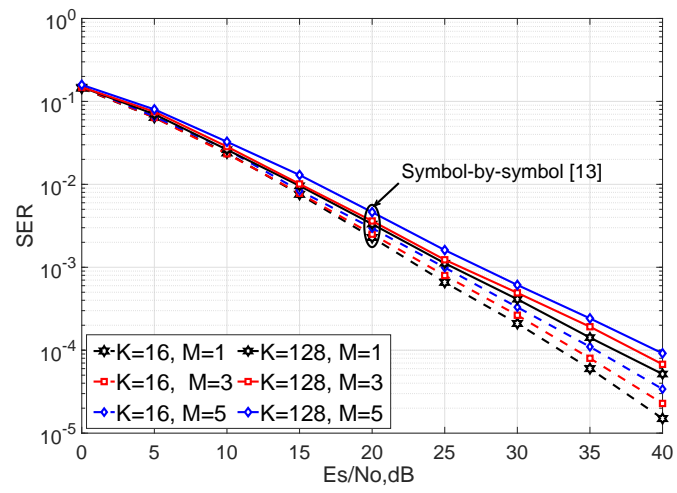

Fig. 1. SER vs. $E_{s} / N_{0}$ for the symbol-by-symbol detection scheme of [13] for GFDM with different $K$ and $M$ in case of BPSK transmission over frequency selective Rayleigh fading channel with $L=9$.

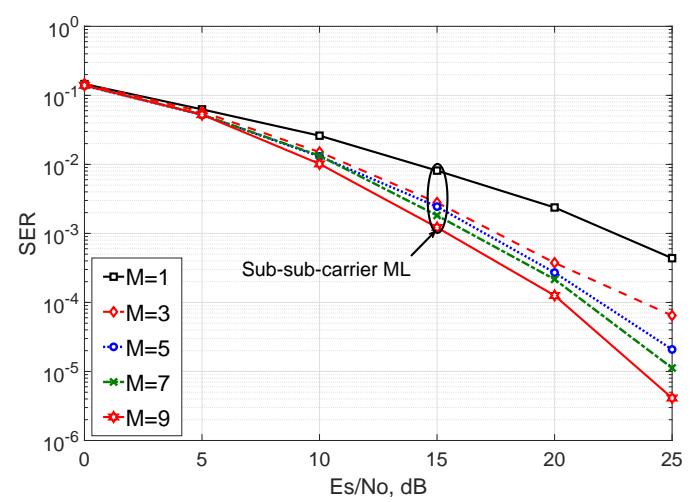

Fig. 2. SER vs. $E_{s} / N_{0}$ for the per-sub-carrier ML detection scheme for GFDM with $K=16$ and different values of $M$ in case of BPSK transmission over frequency selective Rayleigh fading channel with $L=9$.

The weighting matrix $\mathbf{C}_{q M}$ is designed according to the MMSE criterion as

$$
\mathbf{C}_{q M, \mathrm{MMSE}}=\left(\overline{\mathbf{H}}_{q M}^{H} \overline{\mathbf{H}}_{q M}+\sigma^{2} \mathbf{I}_{M}\right)^{-1} \overline{\mathbf{H}}_{q M}^{H},
$$

where $\mathbf{I}_{M}$ is the $M \times M$ identity matrix.

- Block of Symbols Detection, where a decision is simultaneously taken for the block of $M$ symbols transmitted on the same sub-carrier by applying ML principle

$$
\hat{\mathbf{X}}_{q}=\arg \min _{\mathbf{X} \in \mathcal{X}^{M}}\left\|\mathbf{Y}_{q}-\overline{\mathbf{H}}_{q M} \mathbf{X}\right\|^{2},
$$

where $\mathbf{X}$ takes values in the set that contains all the possible $\mathcal{X}^{M}$ transmitted vectors, with $\mathcal{X}$ being the cardinality of the alphabet of the used constellation.

Among these algorithms, it is well known that ML detection is the optimal one in terms of minimizing the probability of error [12]. With ML optimal detection is achieved by means of an exhaustive search over all the combinations of symbols. Although the complexity of ML is quite high, being it proportional to $\mathcal{X}^{M}$, it can be considered as a reference against which to compare the performance of any sub-optimal algorithm.

\section{Computational Complexity}

In this section we only discuss the complexity of the ML approach described in the Sec. III and compare it with

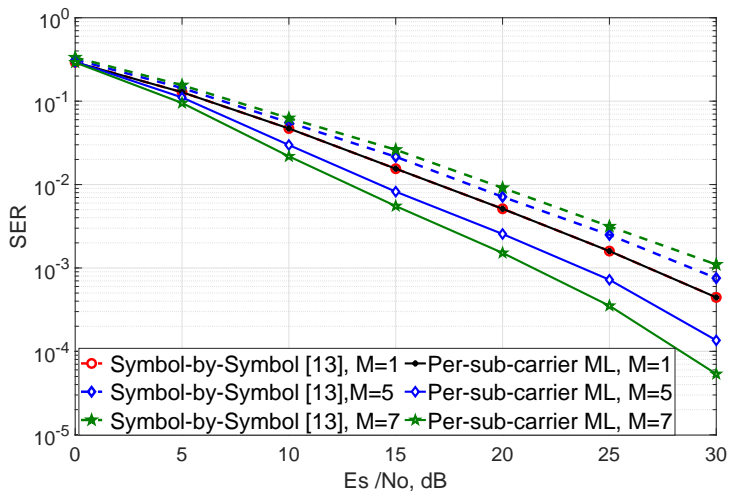

Fig. 3. SER of symbol-by-symbol method of [13] and per-sub-carrier ML detection scheme with $K=16$ and different values of $M$ in case of QPSK transmission over frequency selective Rayleigh fading channel with $L=9$.

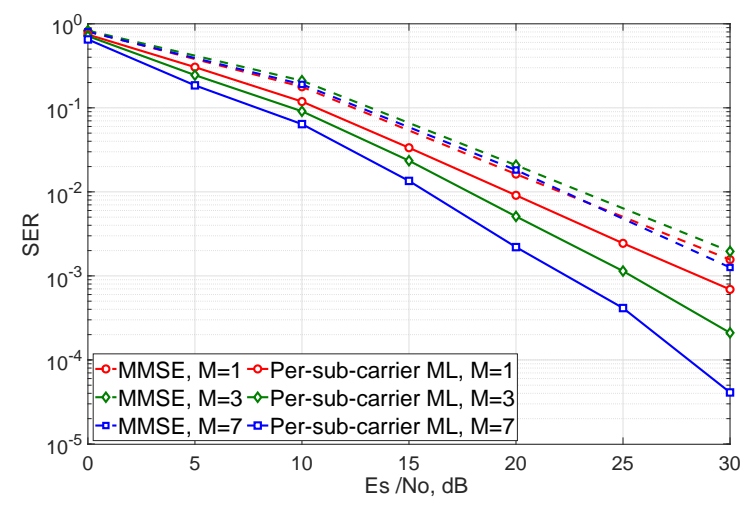

Fig. 4. SER vs. $E_{s} / N_{0}$ for the per-sub-carrier ML detection compred with MMSE with $K=16$ and different values of $M$ in case of 16-QAM transmission over frequency selective Rayleigh fading channel with $L=9$.

that of other approaches available in the literature. This is because, between the two considered detection approaches, ML is the most demanding in terms of number of complex operations, while the complexity of MMSE is very well known being it among the most used schemes. The implementation complexity is evaluated by considering the number of complex multiplications required by each operation performed in the receiver as follows:

- With focus on (12), the first operation consists in evaluating $M$ times a number of $M K$ multiplications to perform the product $\gamma^{*}\left(\langle n-m K\rangle_{N}\right) y(n)$.

- Then the computation of $Y_{q}(m)$ requires an $M K$-point FFT with $\frac{M K}{2} \log _{2}(M K)$ complex multiplications.

- For the ML detection defined by (27) the matrix-vector operation $\overline{\mathbf{H}}_{q M} \mathbf{X}$ requires an exhaustive search over all the combinations of $M$ sub-symbol transmitted on the $q$ th sub-carrier. The total number of required multiplications is $2 \mathcal{X}^{M} M K$.

The overal complexity of the proposed scheme is therefore given by $M^{2} K+M K / 2 \log _{2}(M K)+2 \mathcal{X}^{M} M K$. Table I reports the complexity of different implementations of GFDM receivers, including the proposed one. As it can be easily observed the proposed scheme has a lower complexity as compared to approx. ML symbol-by-symbol detection method proposed in [13] for smaller size of the constellation points. When we increase the constellation size the complexity grows 
TABLE I

COMPLEXITY OF DIFFERENT GFDM RECEIVER IMPLEMENTATIONS

\begin{tabular}{|l|l|}
\hline GFDM Receiver & Number of Complex Multiplications \\
\hline Direct ZF [3] & $(M K)^{2}+M K \log _{2}(M K)+M K+\mathcal{X} M K$ \\
\hline Direct MMSE [3] & $\begin{array}{l}1 / 3(M K)^{3}+(M K)^{2}+M K \log _{2}(M K)+ \\
M K+\mathcal{X} M K\end{array}$ \\
\hline Matched filter + & $M K\left(3 / 2 \log _{2}(M K)+1 / 2 \log _{2} M+I+\right.$ \\
SIC [5] & $\left.1+I_{o}\left(\log _{2} M+1+\mathcal{X}\right)\right)$ \\
\hline Approx. ML [13] & $M K / 2\left(\log _{2}(M K)+\log _{2}(M K)\right)+$ \\
& $(M K)^{2}+2 \mathcal{X} M K$ \\
\hline Proposed scheme & $M K / 2 \log _{2}(M K)+M^{2} K+2 \mathcal{X}^{M} M K$ \\
\hline
\end{tabular}

exponentially due to the exhaustive search required to test all the combinations of $M$ sub-symbols.

\section{Simulation Results}

In this section we present Monte Carlo simulations that show the symbol error rate (SER) performance that can be achieved by using the proposed system model. This allows for the design of several detection algorithms on a per-subcarrier basis. As an example, we consider symbol-by-symbol MMSE and block-symbol ML detection presented in Sec. III. Transmission takes place over the channel defined by (9), where the tap coefficients $h_{i}, i=0,1, \ldots, L-1$, are modeled as independent and identically distributed zero mean complex random variables with average power $\sigma_{i}^{2}=1 / L$, uniform distributed phase in $[0,2 \pi)$, and Rayleigh distributed amplitude. We consider quasi-static assumption where the channel's coefficients remain constant for the entire duration of each block.

Figure 1 shows the SER achieved by the symbol-by-symbol detection method of [13] in case of trasmission over a Rayleigh fading channel with $L=9$ taps for BPSK modulation when $N=16$ and $N=128$ at different values of $M$. As it can be observed, there is a slight degradation in performance due to ISSI, whose effect is higher as $M$ increases. This demonstrates the limits of the symbol-by-symbol method proposed in [13], which does not rely on the knowledge of an exact model for the channel. In constrast, by relying on the system model proposed in this letter, the design of persub-carrier detectors can be done on the basis of the exact channel, as defined by (21). Figure 2 reports the performance achieved by implementing the per-subcarrier ML detection in (27). We see that an increase of $M$ leads to an improvement in SER performance, which confirms the capability of the proposed model in managing ISSI and taking into account the contribution of all the sub-symbols transmitted on the same sub-carrier. Our proposed model is therefore able to take advantage of exact channel knowledge to improve performance in GFDM systems based on Dirichlet pulse shape filtering.

The SER performance obtained for QPSK modulation is reported in Fig. 3 for $K=16$ at different values of $M$. Again a significant improvement in performance is observed in comparison to approx. ML symbol-by-symbol detection of [13]. For the same channel considered in Fig. 3, Fig. 4 shows the SER performance in case of 16-QAM modulation with per sub-carrier detection implemented by MMSE and ML receivers in Sec. III for $K=16$ and different values of $M$. As it can be observed for the MMSE receiver there is a significant performance degradation compared to ML detection even if a proper model of ISSI is available.

\section{CONCLUSION}

In this letter an exact model of ISSI that affects the subsymbols transmitted on the same sub-carrier in GFDM systems is introduced. The model, which is obtained when Dirichlet pulse shaping filter is used, allows for designing detection algorithms, such as MMSE and ML, on a per sub-carier basis. The advantage, in terms of SER performance compared to an existing frequency-domain sub-optimal ML approach recently proposed, is demonstrated by considering transmission over a frequency selective channel, where the optimal ML receiver is designed by using the proposed model. It is worth noting that after deriving the exact system model for GFDM, the analytical evaluation of the average symbol error probability can be done by applying classical techniques to analyze performance in the presence of intersymbol and inter-carrier interference as given, for example, in [15]. However, analtyical performance evaluation is not investigated here due to the lack of space.

\section{REFERENCES}

[1] X. Zhang, et al., "On the waveform for 5G," IEEE Commun. Mag., vol. 54 , no. 11 , pp. 74-80, Nov. 2016.

[2] C. J. Zhang et al., "New waveforms for $5 \mathrm{G}$ networks," IEEE Commun. Mag., vol. 54, no. 11, pp. 64-65, Nov. 2016.

[3] N. Michailow et al., "Generalized frequency division multiplexing for 5th generation cellular networks," IEEE Trans. Comm., vol. 62, no. 9 , pp. 3045-306, Sept. 2014.

[4] M. Matthe, D. Zhang, and G. Fettweis, "Sphere-decoding aided SIC for MIMO-GFDM: Coded performance analysis," in Proc. of ISWCS, pp. 165-169, Sept. 2016.

[5] I. Gaspar, et al., "Low complexity GFDM receiver based on sparse frequency domain processing," in Proc. of VTC Spring, pp. 1-6, Jun. 2013.

[6] F. Schaich and T. Wild, "Waveform contenders for 5G: OFDM vs. FBMC vs. UFMC," in Proc. of ISCCSP, pp. 457-460, May 2014.

[7] A. Kumar and M. Magarini, "Improved Nyquist pulse shaping filters for generalized frequency division multiplexing," in Proc. of LATINCOM, pp. 1-7, Nov. 2016.

[8] C. Tai, B. Su and C. Jia, "Frequency-domain decoupling for MIMOGFDM spatial multiplexing," International Conference on Acoustics, Speech and Signal Processing (ICASSP), May. 2019.

[9] M. Matthé, L. L. Mendes, and G. Fettweis, "Generalized frequency division multiplexing in a Gabor transform setting," IEEE Commun. Lett., vol. 18, pp. 1379-1382, Aug. 2014.

[10] S. Han, et al., "Filter Design for Generalized Frequency-Division Multiplexing," IEEE Trans. on Sig. Proc., vol. 65, no. 7, pp. 1644-1659, Apr. 2017.

[11] M. Matthé,et al., " Influence of pulse shaping on bit error rate performance and out of band radiation of Generalized Frequency Division Multiplexing," IEEE International Conference on Communications Workshops (ICC), June, 2014

[12] H. Artes, D. Seethaler, F. Hlawatsch, "Efficient detection algorithms for MIMO channels: a geometrical approach to approximate ML detection," IEEE Trans. Sig. Proc., vol. 51, no. 11, pp. 2808-2820, Nov 2003.

[13] P. Wei, X. G. Xia, Y. Xiao, and S. Li, "Fast DGT-based receivers for GFDM in broadband channels," IEEE Trans. Comm., pp. 4331-4345, Oct. 2016

[14] M. K. Simon and M.-S. Alouini, Digital Communication over Fading Channels, 2nd ed. New York: Wiley, 2005.

[15] J. Aranda, et al., "Error probability analysis of Nyquist-I pulses in intersymbol and cochannel interference," IEEE Symposium on Computers and Communications (ISCC), June 2018. 